\title{
Um Mapeamento Sistemático para auxiliar na escolha de plataformas EAD para o ensino-aprendizagem de Algoritmos e Programação de Computadores
}

\author{
Jefta Karoline Caldeira, Ana Paula Freitas Vilela Boaventura \\ Departamento de Ciência da Computação - Universidade Federal de Goiás (UFG) - \\ Jataí, GO - Brasil \\ jeftakarolinejtehotmail.com, ana_vilela@ufg.br
}

\begin{abstract}
Currently the Virtual Learning Environments - (AVA) are being used in the teaching - learning process Algorithms and Computer Programming - (APC) in hybrid modes. However, there is no consensus about which platforms are best suited to this purpose. Trying to answer this question, it is this work a systematic mapping databases as IEEE Xplorer and Brazilian Informatics Symposium on Education - SBIE between the years 2011 to 2016. Therefore, the objective of this study was to review the literature to some of the main tools that influence the virtual learning APC. Of the 62 preselected articles remaining 12, of which only 2 articles feature comparison between the different platforms and tools used for the APC of teaching learning.
\end{abstract}

Resumo. Atualmente os Ambientes Virtuais de Aprendizagem - (AVA) estão sendo utilizados no processo de ensino-aprendizagem de Algoritmos e Programação de Computadores - (APC), em modalidades hibridas. Entretanto, não há um consenso sobre quais plataformas são mais adequadas a esta finalidade. Tentando responder essa questão, faz-se neste trabalho um mapeamento sistemático em bases de dados como IEEE Xplorer e Simpósio Brasileiro de Informática na Educação - SBIE, entre os anos de 2011 a 2016. Assim, objetiva-se neste trabalho fazer um levantamento bibliográfico a cerca das principais ferramentas que influenciam no aprendizado virtual de APC. Do total de 62 artigos pré-selecionados, restaram 12, dos quais apenas 2 artigos apresentam comparação entre as diferentes plataformas e ferramentas usadas para o ensino-aprendizagem de APC.

A disciplina de Algoritmos e Programação de Computadores - APC trata-se de uma cadeira introdutória do curso de Ciências da Computação e áreas afins. Embora seja de suma importância para o acadêmico, observa-se um elevado índice de reprovação e ou abandono, conforme Ramos et al (2015), Vihavainen et al. (2014) e Watson e Li (2014).

Para Nobre e Menezes (2002), o desafio do docente desta disciplina está em: i) Reconhecer as habilidades inatas dos alunos; ii) Apresentar técnicas de soluções de problemas; iii) Trabalhar a capacidade de abstração do aluno, tanto na busca de possíveis soluções, quanto na escolha da estrutura de dados, a ser utilizada; iv) Promover a cooperação e colaboração entre os alunos.

Alguns dos conceitos básicos, que são necessários na aprendizagem de algoritmos, são citados na tabela 3, que está baseada nos conceitos de Petry (2005). 
V Congresso Brasileiro de Informática na Educação (CBIE 2016)

Anais do XXVII Simpósio Brasileiro de Informática na Educação (SBIE 2016)

Tabela 3 - Conceitos básicos de aprendizagem de algoritmos

\begin{tabular}{|l|l|}
\hline Conceitos básicos & Definição \\
\hline Raciocínio logico & $\begin{array}{l}\text { Colocar o pensamento em ordem para escolher a } \\
\text { melhor solução para o problema. }\end{array}$ \\
\hline Lógica de programação & $\begin{array}{l}\text { Conseguir entender o problema e escrever uma solução } \\
\text { em forma de algoritmo para resolver tal problema, } \\
\text { enfim conseguir abstrair. }\end{array}$ \\
\hline Linguagem de programação & Conjunto de símbolos finitos, que formam programas. \\
\hline
\end{tabular}

Seja pela natureza abstrata que envolve lógica de programação ou mesmo, pela falta de tempo e afinidade do discente com o curso, o fato é que grande parte dos alunos apresentam dificuldade em assimilar o conteúdo.

No que tange à falta de tempo dos cursistas de APC, uma solução apontada por Haguenauer, et al. (2000) e Araújo, A. M., et al. (2006), está no emprego da modalidade de Ensino a Distância (EAD) e/ou semipresencial (híbrido). Dentre as vantagens é possível elencar a flexibilização de horários e locais para o estudo. Para a concretização desta modalidade, faz-se necessário recorrer aos Ambientes Virtuais de Aprendizagem (AVA's), que em linhas gerais são sistemas computacionais, que coadunando à prática pedagógica, permite a postagem de arquivos, realização de tarefa e promove a interação entre os participantes por intermédio de ferramentas como: fórum, chats, dentre outros. Por definição, um AVA refere-se ao uso de recursos digitais de comunicação, principalmente, através de softwares educacionais via web, que reúnem diversas ferramentas de interação (OLIVEIRA et al., 2001; VALENTINI, SOARES, 2005). E conforme Silva (2011) acrescenta, o AVA é importante, porque reúne recursos que ajudam a diminuir, as possíveis distâncias, entre alunos e professores, quando usados adequadamente, como instâncias mediadoras do processo educativo.

O estudo do emprego do EAD no processo de ensino-aprendizagem de APC é relevante e observa-se elevada quantidade de trabalhos que abordam esta temática. Entretanto, há uma escassez de trabalhos que convirjam no auxílio ao professor (ou tutor) na escolha do AVA que atenda às necessidades dos alunos de Algoritmos e Programação de Computadores. Logo o problema norteador é: Um AVA, adequadamente escolhido, poderá colaborar no processo de ensino-aprendizagem de APC, na modalidade híbrida?

Sendo assim, o objetivo deste artigo é realizar um Mapeamento Sistemático (MS) com o intuito de incluir ou excluir trabalhos que sejam relevantes à elaboração de critérios que permitam escolher plataformas AVA's, voltadas para o ensinoaprendizagem de APC. Como objetivos específicos é possível citar: Apresentar um método para realizar o MS; Elaborar um quadro comparativo com os trabalhos mais relevantes para escolha de AVA; Aprimorar o processo de escolha do AVA. 
V Congresso Brasileiro de Informática na Educação (CBIE 2016)

Anais do XXVII Simpósio Brasileiro de Informática na Educação (SBIE 2016)

\section{Planejamento do Mapeamento Sistemático}

Para a condução deste trabalho foi feita uma adaptação da proposta de Kitchenham (2004), para uma revisão sistemática da literatura. Assim, foram selecionados e catalogados trabalhos que possuíam maior afinidade com a escolha de plataformas AVA's que são empregadas no processo de ensino-aprendizagem de APC, na modalidade híbrida.

Dessa forma, a seguinte questão norteadora foi apontada durante o levantamento do problema norteador: "Quais ferramentas presentes nos AVA's são imprescindíveis para o aprendizado do discente de APC?". O mapeamento pode ser expresso pelo fluxo de atividades expressa na Figura 1:

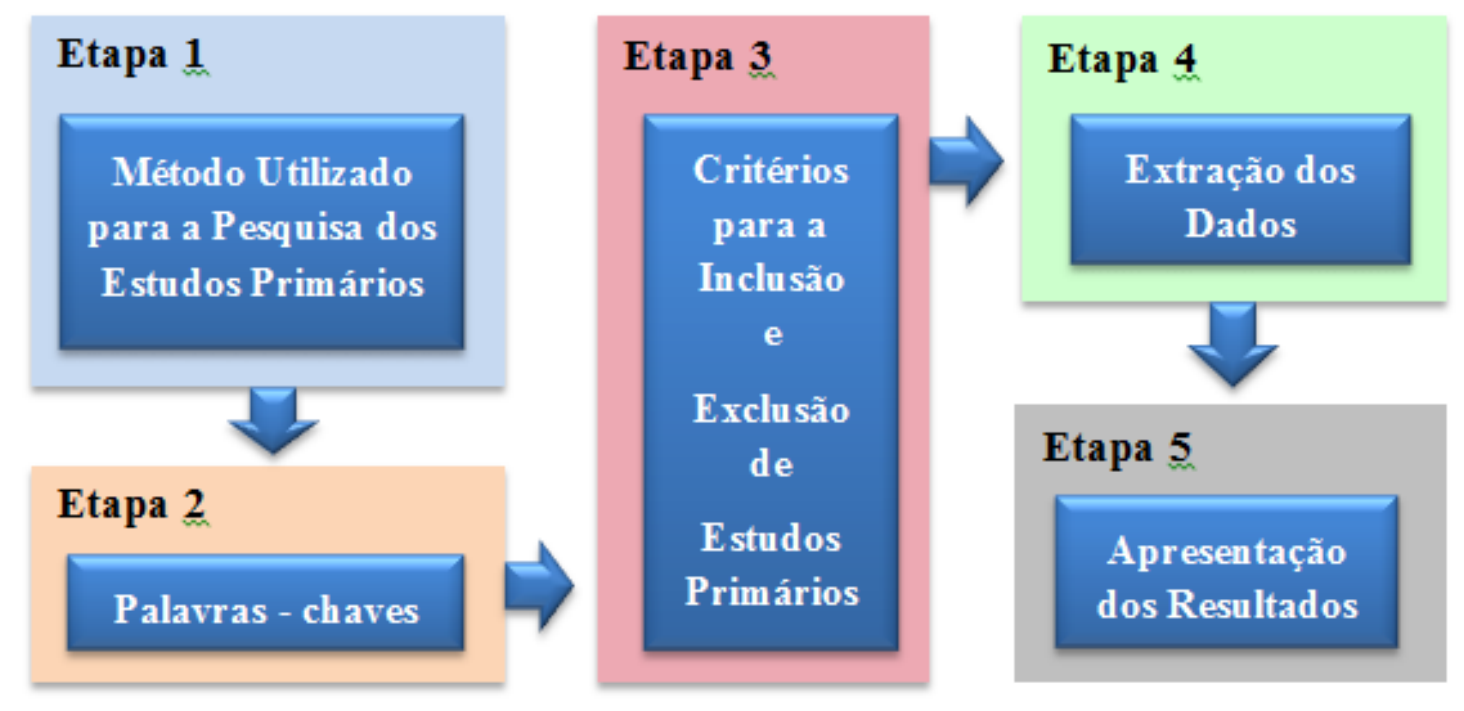

Figure 1. Fluxo de atividades

\subsection{Método Utilizado para a Pesquisa dos Estudos Primários}

Procurando responder à questão norteadora, foi realizada uma busca por artigos nas seguintes bases de dados: IEEE Xplorer, Portal de Periódicos da CAPES, Simpósio Brasileiro de Informática na Educação - SBIE, Revista Magistro, Revista digital CVARICESU e RENOTE - Revista Novas Tecnologias na Educação, de 2011 a 2016.

\subsection{Palavras-chaves}

Para o MS foram utilizados os seguintes strings: EAD, Modalidade híbrida, Plataforma AVA, Ensino - aprendizagem, Aprendizagem, Algoritmos e Programação. Isso foi feito tanto em buscas individuais, como correlacionando duas ou mais strings de busca, como por exemplo: Algoritmos e Programação e Plataforma AVA,

\subsection{Critérios para a Inclusão e Exclusão de Estudos Primários}

Os critérios para inclusão dos estudos primários foram formulados pelas autoras do presente trabalho, com base nas notas de aulas da disciplina de Projeto Final de Curso 2, disciplina ofertada pelo curso de Bacharelado em Ciência da Computação - 
V Congresso Brasileiro de Informática na Educação (CBIE 2016)

Anais do XXVII Simpósio Brasileiro de Informática na Educação (SBIE 2016)

UFG/Regional Jataí. A Tabela 1 apresenta os quatro critérios de inclusão identificados pela primeira coluna, com os respectivos critérios usados para incluir o trabalho.

Tabela 1. Critérios para Inclusão de Estudos Primários

\begin{tabular}{|l|l|}
\hline I1 & Os trabalhos devem estar escritos em inglês, espanhol ou português. \\
\hline I2 & A versão completa dos trabalhos deve estar disponível na web. \\
\hline I3 & $\begin{array}{l}\text { Os trabalhos devem conter como objeto de estudo: O emprego das plataformas } \\
\text { AVA's para ensino - aprendizagem de APC. }\end{array}$ \\
\hline I4 & Trabalhos posteriores ao ano de 2011. \\
\hline
\end{tabular}

A Tabela 2, semelhante à anterior, apresenta os quatro critérios de exclusão identificados pela primeira coluna, com os respectivos critérios usados para excluir o trabalho.

Tabela 2. Critérios para Exclusão de Estudos Primários

\begin{tabular}{|l|l|}
\hline E1 & Os trabalhos que não estavam escritos em inglês, espanhol ou português. \\
\hline E2 & Trabalhos muito antigos. \\
\hline E3 & $\begin{array}{l}\text { Trabalhos que não tinham como objeto de estudo as plataformas AVA's para } \\
\text { ensino - aprendizagem de APC. }\end{array}$ \\
\hline E4 & Trabalhos que não estavam disponíveis na web. \\
\hline
\end{tabular}

\section{Extração dos Dados}

No início foram selecionados sessenta e dois trabalhos, porém após a inclusão e exclusão de trabalhos, chegou-se a um total de doze trabalhos selecionados. Logo em seguida, realizou-se um novo refinamento, com a intenção de eliminar os que não contemplavam a comparação entre plataformas ou não analisavam o uso das plataformas como meio de auxiliar no processo de ensino - aprendizagem em APC. Feito isso, chegou-se a um total de dois trabalhos que foram identificados como trabalhos diretamente relacionados ao tema proposto pelo presente artigo.

\section{Apresentação dos Resultados}

Após o planejamento e execução das etapas do mapeamento sistemático, o resultado da pesquisa convergiu para os dois trabalhos relacionados na Tabela 3. 
V Congresso Brasileiro de Informática na Educação (CBIE 2016)

Anais do XXVII Simpósio Brasileiro de Informática na Educação (SBIE 2016)

Tabela 3. Referências Analisadas neste trabalho.

\begin{tabular}{|c|l|}
\hline ID & \multicolumn{1}{|c|}{ Referência } \\
\hline $\mathbf{4 . 1}$ & Alencar, G. D., et al (2014) \\
\hline $\mathbf{4 . 2}$ & Rosa, M. M.; Giraffa, L. M. M. (2011) \\
\hline
\end{tabular}

As sessões a seguir apresentam uma visão geral dos mesmos.

\subsection{FaceEduc: Análises e Resultados da Adaptação Gráfica do MOODLE Baseada no Facebook}

O trabalho dos autores Alencar, et al (2014) teve como objetivo aprimorar o aprendizado do aluno e reverter o problema de evasão dos alunos nos cursos a distância.

Para isso, Alencar (2014) estruturou o FaceEduc que é a plataforma MOODLE com interface gráfica semelhante a do Facebook, tendo o intuito de cativar e incentivar os alunos. Posteriormente, colocou-os em prática para os alunos da instituição de ensino de nível superior e técnico (IES) no ano de 2013.

Após a intervenção no processo de ensino - aprendizagem, foi feita uma coleta de dados por meio de questionários, durante dois semestres letivos consecutivos com alunos dos semestres de 2013.1 e 2013.2, respectivamente. Em 2013.1, de 793 alunos matriculados, somente 701 responderam ao questionário e em 2013.2, de 382 alunos matriculados, somente 351 responderam ao questionário. Para a análise dos dados foram destacados os seguintes pontos nos questionários: a aceitação dos discentes, a facilidade do uso, o tempo de utilização por semana, acesso no período das férias e a infraestrutura.

Posteriormente, os resultados obtidos entre o MOODLE tradicional e o FaceEduc foram comparados. Como resultados, o autor constatou que em todos os quesitos analisados, a plataforma FaceEduc se destacou em comparação com a plataforma MOODLE. E um dos pontos que merece uma maior atenção é a diferença do tempo de utilização das plataformas no período das férias no qual o FaceEduc se sobressai predominantemente. Observou-se também que houve um aumento de dados armazenados nos servidores, bem como uma elevação no consumo do link de dados.

A conclusão do mesmo é que os alunos se adaptam melhor às tecnologias, quando vão de encontro aos seus interesses e necessidades pessoais. Logo, o uso da ferramenta contribui para o processo de ensino - aprendizagem.

\subsection{O Ensino De Programação De Computadores E EAD: Uma Parceria Possível}

O trabalho de Rosa e Giraffa (2011) identificou que alunos e professores encontram dificuldades no que diz respeito ao ensino - aprendizagem de programação de computadores. Para amenizar o problema, o trabalho teve como objetivo refletir acerca da utilização de ferramentas, na modalidade EAD, no auxilio do ensino de programação. Assim, para alcançar o objetivo do trabalho foi feita uma busca em artigos e livros (da área da educação e da ciência da computação) sobre os pontos em que os alunos encontram uma maior dificuldade no aprendizado da programação e partindo disso, foi feito uma investigação por ferramentas de EAD que pudessem tratar estes problemas. 
V Congresso Brasileiro de Informática na Educação (CBIE 2016)

Anais do XXVII Simpósio Brasileiro de Informática na Educação (SBIE 2016)

Para a busca e a escolha das ferramentas, foram utilizados os quatro princípios básicos em que os ambientes interativos de aprendizado são baseados:

1. O estudante deve construir seu conhecimento;

2. O controle do sistema é feito de forma mais significativa pelo estudante;

3. O sistema é individualizado para cada estudante;

4. O retorno ou feedback é gerado em função da interação do estudante com o ambiente.

Partindo destes quatro pilares, as ferramentas para serem analisadas foram: WebPortugol e Algorithm Learning Internet-based Computer Environment - A.L.I.C.E. A WebPortugol possui uma interface simples e contém apenas as operações necessárias para o desenvolvimento da lógica de programação utilizando a notação de algoritmos, ou seja, o portugol. De acordo com Hostins e Raabe (2007), o WebPortugol trata-se de uma ferramenta eficiente para o que se propõe e ainda encontra-se disponível para livre acesso na web, mediante o preenchimento de uma ficha cadastral. Segundo os autores a característica que apresentou maiores benefícios na ferramenta foi o verificador de algoritmos, pois possibilita aos alunos ampliar o grau de autonomia durante $\mathrm{o}$ desenvolvimento das soluções uma vez que permite aos discentes realizar alguns testes com valores pré-definidos. Somado a isso, não é necessário que os alunos decorem sintaxes em inglês para executar seus algoritmos.

O ambiente A.L.I.C.E, por sua vez, tem o objetivo de completar o conteúdo ministrado em sala de aula. Este sistema dispõe-se de três perfis de usuários: o administrador (gerencia cadastros de alunos, professores, disciplinas, cursos e configuração do sistema), o professor (gerencia o conteúdo instrucional, analisa e acompanha as atividades dos alunos) e o perfil aluno (acessa o conteúdo, interage com os participantes e realiza exercícios). Este ambiente ainda conta com ferramentas de socialização que permitem a troca de mensagens e conhecer o perfil de cada participante. Dentre as funcionalidades é possível citar as ferramentas de detecção de plágio em respostas e um assistente de correção de algoritmo.

Os resultados obtidos foram que com o auxílio dessas ferramentas baseadas em EAD, a tarefa de realizar a programação de computadores se torna mais interessante para o aluno, uma vez que seriam necessárias poucas aulas iniciais para ensinar a lógica e alguns comandos básicos para o aluno poder escrever alguns algoritmos e poder testálos via web. Para os autores o A.L.I.C.E., serve como complemento para o WebPortugol, construído para ser um ambiente de aprendizagem, ele se propõe a auxiliar tanto ao professor quanto ao aluno, pois possui métricas que facilitam ao professor identificar os pontos falhos na aprendizagem, e com isso, retomar alguns conceitos para corrigir estas falhas.

Assim após investigação com artigos e publicações sobre as ferramentas de EAD adequadas ao ensino de programação os autores concluíram que o EAD pode facilitar o aprendizado, fornecendo ferramentas que possibilitam uma maior facilidade no cumprimento das tarefas supracitadas, através do acompanhamento da execução das tarefas, realizadas pelos alunos, caracterizando uma avaliação diagnóstica e contínua, como também um facilitador na observação das habilidades adquiridas durante $o$ 
V Congresso Brasileiro de Informática na Educação (CBIE 2016)

Anais do XXVII Simpósio Brasileiro de Informática na Educação (SBIE 2016)

processo ensino-aprendizagem, identificando os pontos falhos e de sucesso no aprendizado do aluno e do docente.

\section{Conclusão}

O MS apresentado neste trabalho foi inspirado na metodologia de Kitchenham (2004) para revisão sistemática da literatura. Assim, o mesmo foi conduzido de forma manual, fazendo inclusão e exclusão de trabalhos que estavam relacionados com o objeto de estudo - soluções pautadas no EAD que auxiliem no processo de ensino-aprendizagem de algoritmos.

Assim, após uma varredura pelos meios especializados para pesquisa, foi feita uma triagem dos artigos que tinham maior familiaridade com o tema proposto e usando um conjunto de critérios pré-estabelecidos pelos autores do presente trabalho, foram selecionados dois trabalhos, resultando num quadro comparativo de trabalhos relevantes à pesquisa.

De acordo com os dados existem vários métodos que utilizam plataforma AVA's para auxiliar no ensino - aprendizagem de APC. O método mais utilizado são propostas de ambientes construídos especificamente para APC.

Com trabalhos futuros, pretende-se elaborar um conjunto de critérios que auxiliem na escolha de plataforma EAD, para o ensino - aprendizagem de APC, a fim de escolher plataformas que sejam adequadas ao ensino - aprendizagem.

\section{Referências}

Alencar, G. D. , et al. (2014) "FaceEduc: Análises e Resultados da Adaptação Gráfica do MOODLE baseada no Facebook", http://www.lbd.dcc.ufmg.br/bdbcomp/servlet/Trabalho?id=21323, Abril.

Araújo, A. M., et al. (2006) "Estudo comparativo sobre plataformas de EaD baseadas em software livre". SBIE.

Haguenauer, C.J., et al (2000) "International View on Engineering Education", ed da Pontifícia Universidade Católica do Rio de Janeiro, em co-autoria com Luis Carlos S. do Carmo et al, Rio de Janeiro.

Hostins, H., Raabe, A. L. A. (2007) "Auxiliando a Aprendizagem de Algoritmo com a Ferramenta WebPortugol”, XV Workshop sobre Educação em Computação - WEI, Rio de Janeiro, RJ, Brasil.

Kitchenham, B. (2004) "Procedures for performing systematic reviews", Keele, UK, Keele University, 33(2004):1-26.

Nobre, I. A. M.; Menezes, C. S. (2002) "Suporte à Cooperação em um Ambiente de Aprendizagem para Programação (SAmbA)", XII Simpósio Brasileiro de Informática na Educação - SBIE - UNISINOS, pp. 337-347.

Oliveira, C.C., et al. (2001) "Ambientes Informativos de Aprendizagem: produção e avaliação de software educativo", Campinas: Editora Papirus.

Petry, P. G. (2005) "Um sistema para o ensino e aprendizagem de algoritmos utilizando um companheiro de aprendizagem colaborativo", Florianópolis: UFSC. 
V Congresso Brasileiro de Informática na Educação (CBIE 2016)

Anais do XXVII Simpósio Brasileiro de Informática na Educação (SBIE 2016)

Ramos, V. et al. (2015) “A Comparação da Realidade Mundial do Ensino de Programação para Iniciantes com a Realidade Nacional: Revisão sistemática da literatura em eventos brasileiros", In: Anais do Simpósio Brasileiro de Informática na Educação, p. 318.

Rosa, M. M.; Giraffa, L. M. M. (2011) "O ensino de programação de computadores e EAD: uma parceria possível”, Porto Alegre - RS, Março.

Silva, I. M. M. (2011) "Interfaces digitais na educação a distância: das salas de aula aos ambientes virtuais de aprendizagem", Revista Digital da CVA-RICESU, v. 7, n. 25.

Valentini, C. B., Soares, E. M. S. (2005) "Aprendizagem em Ambientes Virtuais: compartilhando ideias e construindo cenários", Caxias do Sul: EDUCS.

Vihavainen, A. et al. (2014) "A Systematic Review of Approaches for Teaching Introductory Programming and Their In- fluence on Success", In Proceedings of the Tenth Annual Conference on International Computing Education Research, ICER '14, pages 19-26, New York, NY, USA. ACM.

Watson, C., Li, F. W. (2014) "Failure Rates in Introductory Programming Revisited", In Proceedings of the 2014 Conference on Innovation \& Technology in Computer Science Education, ITiCSE '14, pages 39-44, New York, NY, USA. ACM. 